\title{
Characterization of Refractive Index Change Induced by Femtosecond Laser in Lithium Niobate
}

\author{
Jian-Guan Hua, Feng Yu, Zhen-Nan Tian, Yan-Hao Yu, and Yong-Sen Yu* \\ State Key Laboratory on Integrated Optoelectronics, College of Electronic Science and Engineering, \\ Jilin University, Changchun 130012, Peoples Republic of China \\ *E-mail: yuys@jlu.edu.cn
}

\begin{abstract}
When femtosecond laser is focused into optical materials, their refractive index can be changed. Then various important optical elements can be fabricated by this technology. Herein, we report a simple and practical method to measure the refractive index changes induced by femtosecond laser in optical materials. By fabricating the gratings in a Z-cut $\mathrm{LiNbO}_{3}$ crystal and measuring the ratio of grating's different diffraction orders' energy, the refractive index change $\left(3.4 \times 10^{-3}\right)$ in $\mathrm{LiNbO}_{3}$ crystal by femtosecond laser irradiation was obtained. This approach represents a simple way for measuring the refractive index change induced by femtosecond laser in a variety of transparent materials like sapphire, quartz and glass etc. In addition, this method would find the great technological importance in fabrication of laser written refractive index structures such as lens, DOEs and other optical structures.
\end{abstract}

DOI: 10.2961/jlmn.2017.03.0006

Keywords: femtosecond laser, refractive index, gratings, diffraction, optical materials

\section{Introduction}

Recently, buried micro-optical elements have gained significant attention in scientific research and technological applications owing to their distinctive advantages. They remain unaffected by dust scratches, rubs and vibrations. Besides, the influence of any external environment is extremely little. Instead, the surface optical elements are entirely different. The diffraction efficiency of surface optical element decreases dramatically at the design wavelength when the external environment refractive index changes. Based on the aforementioned advantages of buried elements, the buried waveguide [1-4], grating [5-7], and zone plate $[8,9]$ have been fabricated, which showed excellent optical properties. Almost all the buried optical elements are fabricated by the photorefractive effect $[10,11]$. When a focused laser beam scans in a transparent material, the refractive index will change on account of the effect [12]. Then when a light penetrates the area scanned by laser, the phase delay can be modulated, resulting from the change of the refractive index. The phase difference is equal to the product of the optical path and the refractive index change, which affects the optical properties directly. Therefore, it is very urgent to quantitatively measure the refractive index change.

Several methods have been proposed to characterize the refractive index change, such as the transmitted-beam nearfield method [13], two-beam interferometric method [1415], and digital holographic method [16] etc. These methods above aim at particular size optical elements for different applications. Using the relative intensities of various orders is a straight forward way to measure refractive index changes [17]. It has been reported that first order diffraction efficiency of thin gratings estimated refractive index change [18]. However, the experiment used the polymer materials with poor processing accuracy, and can't be used for buried micro-optical components of the design. So far, there is no effective method for tiny photorefractive constructions. In this work, we adopt a novel method based on the scalar diffractive theory to character the refractive index change, which can apply to the diffraction optical element design. We fabricated a series of buried micro-optical gratings by femtosecond laser direct writing technology [19-21] which were buried inside of lithium niobate crystal. In other words, we used a focused femtosecond laser beam to generate a series of periodically arranged stripsowing to multi-photon absorption effect under the upper surface of the crystal $[22,23]$. Then, the refractive index change has been figured out by measuring the energy ratio of the grating's diffraction orders. The method for measuring the refractive index change has many advantages such as the simple experimentation process, low requirements for processing technology and materials, the relatively high precision of measurement results. It is an important guide to develop the laser induced refractive index changed optical elements.

\section{Theory and experiments}

The optical grating is an important optical element and is widely used in optical communication and sensor fields [24]. When a light beam passes through a grating, its energy can be allocated to the diffraction spots with various orders. The grating equation is given by

$$
d \sin \theta=m \lambda
$$

where $d$ is the grating's period, $\theta$ is the angle of the diffracted ray, $m$ is the diffraction order, and $\lambda$ is the wavelength of incident beam. It is obvious from the grating equation that the angle between each order is related to $\lambda$ and $d$. For rectangle waveguide gratings, the equation for the efficiency of each diffraction order can be written as

$$
\begin{aligned}
& \eta_{0}=1-2 \rho(1-\rho)(1-\cos \Delta \phi) \\
& \eta_{m>0}=\frac{(1-\cos 2 m \pi \rho)(1-\cos \Delta \phi)}{m^{2} \pi^{2}}
\end{aligned}
$$


where $\eta_{0}$ and $\eta_{\mathrm{m}}>0$ represent the diffraction efficiency of 0 order and $m$ order respectively [25-27]. In the last equation, $\rho$ is the duty ratio of grating and $\Delta \phi$ is the phase delay of light wave. The diffraction efficiency equation makes clear that the energy of each order depends on $\rho$ and $\Delta \phi$. Moreover, the phase delay $\Delta \phi$ can be obtained by measuring the diffraction efficiency $\eta$ on the premise of obtaining the duty ratio $\rho$. Besides, phase delay $\Delta \phi$ is given by

$$
\Delta \phi=\frac{2 \pi \Delta n h}{\lambda}
$$

where $\Delta n$ is the refractive index change and $h$ is the grating's thickness. It is evident from the equation that the phase $\Delta \phi$ is related to $\Delta n$ and $h$. Therefore, the phase delay can be gained by measuring the energy ratio of the grating's diffraction orders with various orders, and the refractive index change can be figured out under the condition of known thickness.

When femtosecond laser is focused into the lithium niobate crystals, the refractive index at the laser focus can be changed. The laser energy is rarely absorbed with low pulse energy. With the pulse energy exceeding the damage threshold of the crystal, the crystal will appear very strong nonlinear light absorption. The absorption of energy causes the local ionization of the crystal to produce plasma, and the crystal structure changes eventually cause permanent damage. It is generally believed that the main reason of permanent damage is the high temperature and the generation of high density plasma [28]. And the magnitude of the refractive index change is closely related to the laser energy. So the various important optical elements can be fabricated by this technology. The optical gratings with different duty ratios have been fabricated in $\mathrm{Z}$-cut $\mathrm{LiNbO}_{3}$ crystal by femtosecond laser direct writing technology, which is an important and irreplaceable method to fabricate threedimensional buried micro-optical elements [29-33]. The laser source (Spectra Physics Solstice) delivered the laser of $800 \mathrm{~nm}$ wavelength, 120 fs pulse width and $2500 \mathrm{~Hz}$ repetition rate. In order to improve the processing accuracy and achieve two-photon absorption effect, a femtosecond laser of $400 \mathrm{~nm}$ wavelength was obtained by a second harmonic generation system. The laser was tightly focused inside a $500 \mu \mathrm{m}$ thick $\mathrm{LiNbO}_{3}$ crystal by a high numerical aperture objective (NA=0.85, $\mathrm{x} 80$ ) and scanned once by a dual galvanometer scanning system. The scanning speed of femtosecond laser is $200 \mu \mathrm{m} / \mathrm{s}$. The grating duty ratio is controlled by scanning line stacking with $200 \mathrm{~nm}$ spacing. The $\mathrm{LiNbO}_{3}$ crystal was fixed on a piezoelectric stage (PI P-721SL2). The piezoelectric stage controlled the scanning depth of the laser focus to control the depth of processing, and its precision could reach $1 \mathrm{~nm}$. In the machining process, the laser power was $250 \mu \mathrm{W}$, which was measured in front of the focusing objective. Above all, the freewill highprecision three-dimensional structure can be achieved by employing the piezoelectric stage and the dual galvanometer scanning system.

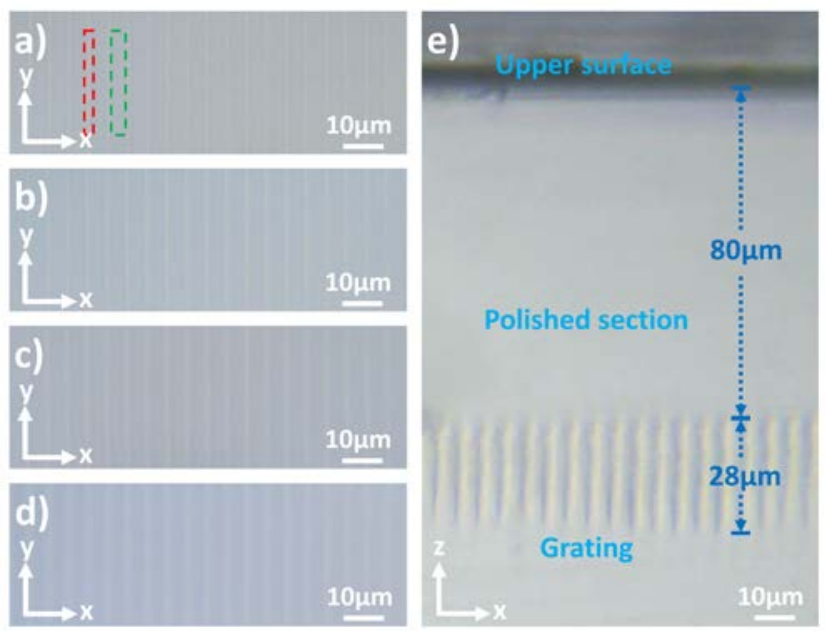

Fig.1 Optical microscope images of the gratings. The duty ratios are (a) 2:23, (b) 1:4, (c) 7:18 and (d) 2:3, respectively. (e) Cross section of the grating.

The images of the optical gratings captured with $\mathrm{x} 40$ micron objectives lens are shown in the figure 1(a)-(d). The period of the gratings is $5000 \mathrm{~nm}$. The figure 1 (a)-(d) show the different gratings whose duty ratios are 2:23, 1:4, 7:18, 2:3 respectively. Both the length and width of the grating structures are $100 \mu \mathrm{m}$. The thickness of the refractive index changed area is $28 \mu \mathrm{m}$. The area marked in the red frame is the structure scanned by femtosecond laser and it appears as bright stripes. The area marked in the green frame is the structure without process and it appears as dark stripes. The figure 1(e) shows the cross section of the grating, which was polished by sandpaper. The grating is buried under the surface of the crystal with the depth of $80 \mu \mathrm{m}$, so its optical performance can't be affected by external environment. From the images, it is obvious that the periodic striped pattern is regular. Besides, the obvious color differences between the two areas can be clearly distinguished. In fact, the color difference is due to the difference of the material's refractive index. The refractive index change modulates the phase of light wave and results in the phase delay. The phase delay of the periodic stripes generates the grating diffraction effect, distributing the energy of a beam to each order. The variation of refractive index determines energy of each diffraction order, and it can be obtained by measuring the energy ratio of diffraction orders.

\section{Results and discussion}

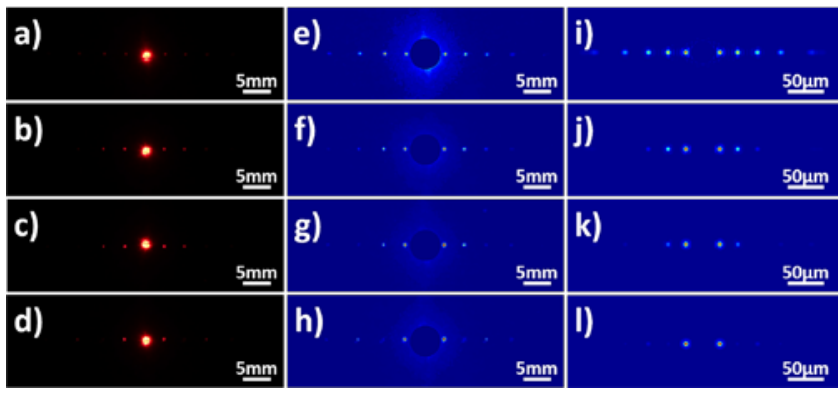

Fig.2 Optical photographs of grating diffraction patterns, the duty ratios are (a) 2:23, (b) 1:4, (c) 7:18 and (d) 2:3, respectively; (e)-(h) Processing of (a)-(d); (i)-(l) Theoretical simulations of grating diffraction patterns. 
Light intensity can be allocated to the diffraction spots with various orders while a beam of light pass through a grating. The light intensity of diffraction spots is closely related to duty ratio and phase delay of the incident wave. Moreover, the phase delay is determined by refractive index change in the condition of a fixed grating thickness, hence the refractive index change can be obtained by measuring the light intensity of diffraction spots. In the experiment, we conducted optical diffraction testing on the fabricated optical gratings. A He-Ne laser of $633 \mathrm{~nm}$ wavelength was adopted as the illuminant to transmit light, and obvious diffraction spots with remarkable orders were obtained. The incident laser was perpendicular to the Z-cut $\mathrm{LiNbO}_{3}$ crystal surface, so the refractive index of the ordinaire light was same as the refractive index of the extraordinaire light in the crystal. We used optical camera to take photos of the diffraction grating patterns with different duty ratios, as in the figure 2(a)-(d). It is easy to find that the energy distribution differences between the orders are quite striking for different duty ratios. For the figure 2(a), the energy intensity decreases with the diffractive order increasing, while for the figure 2(d), the energy of 1st order and 3rd order is high and the energy of 2nd order and 4th order is relatively weak. In order to remove the effects of zero order, the meaningless zero order spot was eliminated, as in the figure 2(e)-(h). Furthermore, the theoretical grating diffraction patterns with the same parameters have been simulated by optical wave propagation based on the Fresnel diffraction theory, as in the figure 2(i)-(l). Apparently, the diffraction patterns of the test results are identical with those of theoretical simulation results. Thus, these optical gratings fabricated by femtosecond laser direct writing technology have an excellent optical performance. a)

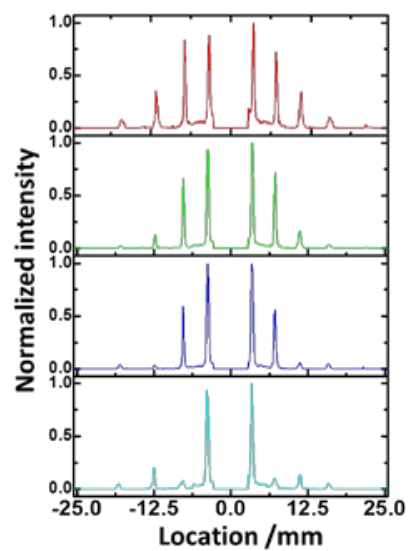

b)

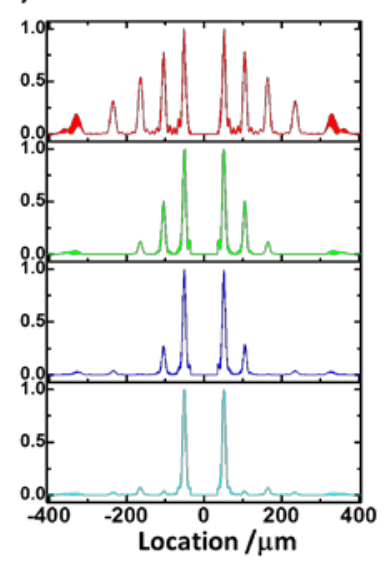

Fig.3 Energy distributions of diffraction patterns. (a) The experimental test results, the duty ratios are 2:23,1:4,7:18 and 2:3, respectively; (b) The theoretical simulation results.

The energy of diffraction orders and the duty ratio of grating hold a relationship. To present that relationship, we have extracted energy from the diffraction patterns of experiment and simulation (figure 2(e)-(l)). The relation curves between energy intensity and location have also been plotted, as in the figure 3. The figure 3(a) describes the experimental test results. The figure 3(b) describes the theoretical simulation results. Moreover, experimental results and simulation results are highly identical. Specially, for the duty ratio of 1:4, the energy of the 1st diffraction order is probably two times as the energy of the 2nd diffraction order, while the energy of the 3rd diffraction order is close to zero. They are identical with the simulation results. Furthermore, it is evident that the gratings with different duty ratios have different energy distributions. Accordingly, the results support that the energy of each diffraction order has a close relation with the duty ratio.

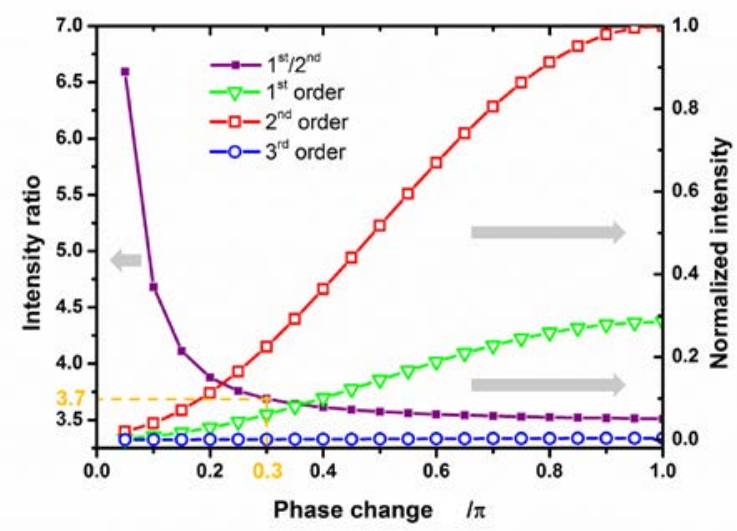

Fig.4 Theoretical relation curves of the energy and the ratio along with the phase change, and the duty ratio is 7:18.

Besides the duty ratio of grating, the energy of each diffraction order is also related to phase delay of the optical wave. For the duty ratio of $7: 18$, the theoretical relation curves between the energy of each diffraction order and phase change are shown in the figure 4 . When the phase change is close to zero, the energy is also close to zero. When the phase change is $0.5 \pi$, the energy becomes maximized. It's easy to find that the 1st diffraction order energy changes quickly along with phase change, the 2nd diffraction order energy changes slowly, while the 3rd diffraction order energy nearly remains unchanged. Moreover, we have selected the 1st order and the 2nd order, and the varying relationship of their energy ratios with phase changes is shown by the line. It is clear in the figure 4 that the energy ratio becomes less and less in one-to-one match along with improvement of phase change. In general, the energy of grating's diffraction order is determined by the duty ratio and phase delay.

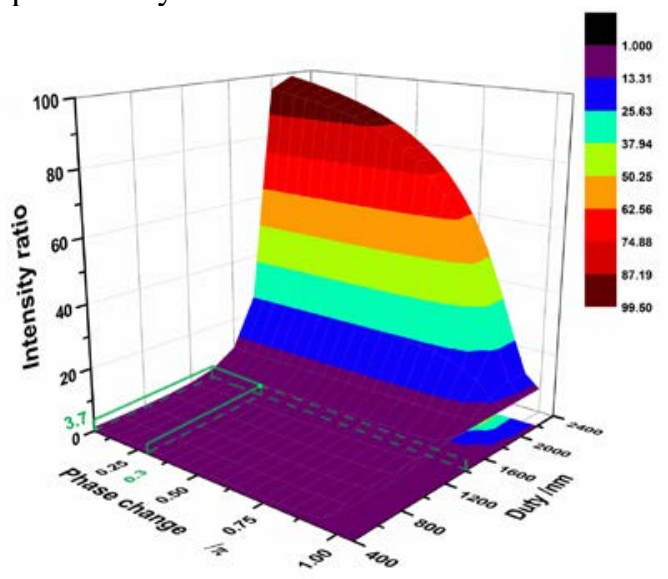

Fig.5 Theoretical relation surface of the energy ratio along with the duty ratio and the phase change. 
In order to show the relationship between the energy ratio, the duty ratio and the phase change, the varying relationships of the energy ratios with phase changes for different duty ratios have been simulated, as in the figure 5 . That is, the figure 5 describes the theoretical relation surface of the energy ratio between the 1st diffraction order and the 2nd diffraction order along with the duty ratio and phase change. Therefore, for a grating with an arbitrary duty ratio, the phase change can be obtained from the relation surface by measuring the energy ratio between the 1st diffraction order and the 2nd diffraction order. In the experiment, the energy of each diffraction order has been measured by a laser power meter for the duty ratio of 7:18, and the thickness of the grating has been obtained by polishing the cross section of the grating. The energy ratio between the 1st diffraction order and the 2nd diffraction order has been figured out, which is 3.69. Then the phase change of $0.3 \pi$ has been obtained from the theoretical relation surface. With the grating's thickness of $28 \mu \mathrm{m}$, refractive index change $\left(3.4 \times 10^{-3}\right)$ has been obtained. Various methods to measure the refractive index change have been reported, and the results are mostly of $10^{-3}$ magnitude, which change along with the experiment parameters [13, 34, 35]. However, most of these methods are very complicated. This method can get the accurate refractive index change. And almost all of the buried optical elements can be designed with the refractive index change. Hence, the method has a guiding significance for the improvement of the refractive index changed optical elements.

\section{Conclusions}

In conclusion, this letter describes a method for measuring the refractive index change. First, we fabricated the buried gratings with different duty ratios in $\mathrm{LiNbO}_{3}$ crystal and obtained their diffraction patterns. Besides, the relationship between the energy ratio of diffraction orders and the phase delay have been simulated for a certain duty ratio, which is strict one-to-one match. Finally, the refractive index change has been obtained by measuring the energy ratio of the diffraction spots. This method is utterly significant for the development of the laser induced refractive index changed optical elements.

\section{Acknowledgments}

The author thanks the supporting by the National Natural Science Foundation of China (Grant No. 91423102, 61590930, 61137001, 91323301 and 61435005).

\section{References}

[1] R. R. Thomson, S. Campbell, I. J. Blewett, A. K. Kar and D. T. Reid: Appl. Phys. Lett., 88, (2006) 111109.

[2] S. G. Zhang, J. H. Yao, Q. Shi, Y. G. Liu, W. W. Liu, Z. C. Huang, F. Y. Lu and E. N. Li: Appl. Phys. Lett., 92, (2008) 231106.

[3] N. Pavel, G. Salamu, F. Voicu, F. Jipa, M. Zamfirescu and T. Dascalu: Laser Phys. Lett., 10, (2013) 095802.

[4] J. Lv, Y. Cheng, Q. Lu, J. R. V. Aldana, X. Hao and F. Chen: Opt. Mater., 57, (2016) 169.

[5] D. Paipulas, R. Buivydas, S. Juodkazis and V. Mizeikis: J. Laser. Micro Nanoen., 11, (2016) 246.

[6] M. Beresna and P. G. Kazansky: Opt. Lett., 35, (2010) 1662.
[7] S. Kroesen, W. Horn, J. Imbrock and C. Denz Opt. Exp., 22, (2014) 23339.

[8] A. Puisys and D. Paipulas: J. Laser. Micro Nanoen., 10, (2015) 96.

[9] I. B. Sohn, M. S. Ahsan, Y. C. Noh, H. K. Choi, J. T. Kim and M. J. Ko: Opt. Eng., 53, (2014) 688.

[10] O. Beyer, I. Breunig, F. Kalkum and K. Buse: Appl. Phys. Lett., 88, (2006) 051120.

[11]E. G. Gamaly, S. Juodkazis, V. Mizeikis, H. Misawa, A. V. Rode, W. Z. Krolikowski and K. Kitamura: Curr. Appl. Phys., 8, (2008) 416.

[12]E. G. Gamaly, S. Juodkazis, V. Mizeikis, H. Misawa, A. V. Rode and W. Krolikowski: Phys. Rev. B, 81, (2010) 054113.

[13] L. Gui, B. X. Xu and T. C. Chong: IEEE Photon. Technol. Lett., 16, (2004) 1337.

[14] M. Ishige, M. Aketagawa, T. B. Quoc and Y. Hoshino: Meas. Sci. Technol., 20, (2009) 084019.

[15] D. Wu, S. Z. Wu, Q. D. Chen, Y. L. Zhang, J. Yao, X. Yao, L. G. Niu, J. N. Wang, L. Jiang and H. B. Sun: Adv. Mater., 23, (2011) 545.

[16] J. W. Zhang, J. L. Di, Y. Li, T. L. Xi and J. L. Zhao: Opt. Exp., 23, (2015) 27328.

[17] P. J. Scully, D. Jones and D. A. Jaroszynski: J. Opt. A: Pure Appl. Opt., (2003) S92.

[18] L. Ye, W. Perrie, O. J. Allegre, Y. Jin, Z. Kuang, P. J. Scully, E. Fearon, D. Eckford, S. P. Edwardson and G. Dearden: Laser Phys., 23, (2013) 126004.

[19] M. Malinauskas, A. Zukauskas, S. Hasegawa, Y. Hayasaki, V. Mizeikis, R. Buividas and S. Juodkazis: Light-Sci. Appl., 5, (2016) e16133.

[20] S. Quan, K. Ueno, H. Yu, A. Kubo, Y. Matsuo and H. Misawa: Light-Sci. Appl., 2, (2013) e118.

[21]B. B. Xu, L. Wang, Z. C. Ma, R. Zhang, Q. D. Chen, C. Lv, B. Han, X. Z. Xiao, X. L. Zhang, Y. L. Zhang, K. Ueno, H. Misawa and H. B. Sun: Acs Nano, 8, (2014) 6682.

[22] L. J. Mv, Y. Z. Cheng, J. R. V. Aldana, X. T. Hao and F. Chen: J. Lightwave Technol., 34, (2016) 3587.

[23] J. M. Lv, Y. Z. Cheng, W. H. Yuan, X. T. Hao and F. Chen: Opt. Mater. Exp., 5, (2015) 1274.

[24] T. Lei, M. Zhang, Y. R. Li, P. Jia, G. N. Liu, X. G. Xu, Z. H. Li, C. J. Min, J. Lin, C. Y. Yu, H. B. Niu and X. C. Yuan: Light-Sci. Appl., 4, (2015) e257.

[25]E. Popov and M. Neviere: J. Opt. Soc. Am. A., 17, (2000) 1773.

[26] M. G. Moharam and T. K. Gaylord: J. Opt. Soc. Am. A., 72, (1982) 1385.

[27]L. Li: J. Opt. Soc. Am. A., 10, (1993) 2581.

[28]B. N. Chichkov, C. Momma, S. Nolte and A. Tunnermann: Appl. Phys. A., 63, (1996) 109.

[29] M. Malinauskas, A. Zukauskas, S. Hasegawa, Y. Hayasaki, V. Mizeikis, R. Buividas and S. Juodkazis: Light-Sci. Appl., 5 (2016) e16133.

[30] J. T. Lin, Y. X. Xu, Z. W. Fang, M. Wang, J. X. Song, N. W. Wang, L. L. Qiao, W. Fang and Y. Cheng: Sci. Rep., 5, (2015) 5:8072.

[31] Y. Cheng, K. Sugioka, M. Masuda, K. Shihoyama, K. Toyoda and K. Midorikawa: Opt. Exp., 11, (2003) 1809. 
[32]Z. F. Deng, Q. Yang, F. Chen, X. W. Meng, H. Bian, J. L. Yong, C. Shan and X. Hou: Opt. Lett., 40 (2015) 1928.

[33] Y. Liao, J. L. Ni, L. L. Qiao, M. Huang, Y. Bellouard, K. Sugioka and Y. Cheng: Optica, 2, (2015) 329.

[34] R. Berlich, J. Choi, C. Mazuir, W. V. Schoenfeld, S. Nolte and M. Richardson: Opt. Lett., 37, (2012) 3003.

[35] A Saad, Y Cho, F Ahmed and M B G Jun: Materials, 9 (2016) 941.

(Received: July 25, 2017, Accepted: October 3, 2017) 